\title{
Mutation screening of the medium-chain acyl-CoA dehydrogenase (MCAD) and the ornithine transcarbamylase (OTC) genes by multiplex PCR amplification and sequencing
}

\author{
Michael P. Horn", Gabriela Mäder-Heinemann², \\ Gisela Andrey ${ }^{2}$ and Carlo R. Largiadèr ${ }^{1,2, *}$ \\ ${ }^{1}$ Molecular Diagnostics DOLS, Inselspital, Bern \\ University Hospital and University of Bern, Bern, \\ Switzerland \\ ${ }^{2}$ Institute of Clinical Chemistry, Laboratory of \\ Molecular Diagnostics, Bern University Hospital and \\ University of Bern, Inselspital, Bern, Switzerland
}

\begin{abstract}
Background: Sequencing based mutation screening assays of genes encompassing large numbers of exons could be substantially optimized by multiplex PCR, which enables simultaneous amplification of many targets in one reaction. In the present study, a multiplex PCR protocol originally developed for fragment analysis was evaluated for sequencing based mutation screening of the ornithine transcarbamylase $(O T C)$ and the medium-chain acyl-CoA dehydrogenase $(M C A D)$ genes.
\end{abstract}

Methods: Single exon and multiplex PCR protocols were applied to generate PCR templates for subsequent DNA sequencing of all exons of the OTC and the MCAD genes. For each PCR protocol and using the same DNA samples, 66 OTC and 98 MCAD sequence reads were generated. The sequences derived from the two different PCR methods were compared at the level of individual signal-to-noise ratios of the four bases and the proportion of highquality base-signals.

Results: The single exon and the multiplex PCR protocol gave qualitatively comparable results for the two genes.

Conclusions: Many existing sequencing based mutation analysis protocols may be easily optimized with the proposed method, since the multiplex PCR protocol was successfully applied without any re-design of the PCR primers and other optimization steps for generating sequencing templates for the OTC and MCAD genes, respectively.

Clin Chem Lab Med 2009;47:56-9.

*Corresponding author: Carlo R. Largiadèr, Institute of Clinical Chemistry, Laboratory of Molecular Diagnostics, Bern University Hospital and University of Bern, Inselspital, Sahli-Haus 2, 114, 3010 Bern, Switzerland

Phone: +41-31-6329545, Fax: + 41-31-6320310,

E-mail: carlo.largiader@insel.ch

Received July 22, 2008; accepted October 7, 2008;

previously published online December 5, 2008
Keywords: DNA sequencing; multiplex PCR; mutation screening.

Multiplex PCR is a variant of PCR enabling simultaneous amplification of many targets of interest in one reaction by using more than one pair of primers. Although the advantage of this technique is obvious in optimizing sequencing based mutation screening assays of genes encompassing a large number of exons, only very few such assays have been developed so far. This is mainly because multiplex assays have generally been tedious and time-consuming to establish, requiring lengthy optimization procedures.

DNA sequencing is a gold standard for screening for known and unknown disease causing mutations in many genes, such as the medium-chain acyl-CoA dehydrogenase (MCAD; RefSeq: NM_000016.2) and the ornithine transcarbamylase (OTC; RefSeq: NM_000531.3). The so-far known mutations are distributed over all exons of these genes, and there are no mutation detection kits available. Until now, all exons of these genes have been screened for mutations with a rather time-consuming procedure consisting of single-exon PCR-amplification, purification, and sequencing.

$M C A D$ catalyzes the $\beta$-oxidation of fatty acids, whereas OTC is an enzyme of the urea cycle and catalyzes the formation of citrulline and inorganic phosphate from carbamyl phosphate and ornithine. Mutations in these genes may result in deficient enzymes, which in the case of MCAD deficiency can result in catabolic stress with some patients dying due to catastrophic neurological damage during decompensation with hypoketotic hypoglycemia (1). OTC deficiency is the most frequently inherited defect of the urea cycle and has an estimated prevalence of one in 14,000 (2). Complete OTC deficiency results in acute hyperammonemia in the first year of life and as a consequence has toxic effects on the brain. In severe cases, hyperammonemia can result in coma, brain edema, and death due to vascular compromise or brain herniation. MCAD is located on chromosome $1 \mathrm{p} 31$ and is encoded by 12 exons separated by 11 introns, and the gene coding for OTC is located on the short arm of chromosome $X$, spans $73 \mathrm{~kb}$ and contains 10 exons and nine introns. So far, 72 mutations have been described in the MCAD gene (www. hgmd.cf.ac.uk), also 341 mutations and 29 non-disease-causing mutations and polymorphisms have been described in the OTC gene [reviewed in ref. (2)]. 
Screening for mutations in the genes encoding for $M C A D$ or OTC is applied early in life, when symptoms of metabolic disorders are diagnosed. In addition, if familiar history is known, mutation screening is often applied as a prenatal molecular diagnostic test based on chorionic villi sampling.

Here, we describe a rapid and reliable method for generating templates for the sequencing of all exons of the OTC and MCAD genes, respectively. This method is based on multiplex PCR reactions using the same primers as for the amplification of single exons (Table 1). For evaluation of the multiplex PCR, we sequenced the PCR products of all exons from both genes with DNA samples from three or five donors, respectively. The quality of these sequences was compared with results obtained from validated standard protocols based on single-exon PCR, used in our laboratory for routine diagnostics.

To reduce the workload and risk of (pipetting-) errors, primer master-mixes containing forward and reverse primers of single exons according to Table 1 were prepared. Once tested, these master-mixes were used in multiplex PCR for the amplification of the different exons serving as sequencing templates. Even though the set-up of multiplex PCR reactions might be very difficult (3), we were successful in setting up multiplex PCR reactions, as shown in Figure 1. In the multiplex PCR for MCAD (Figure 1A) or OTC (Figure 1B), the same amplicons were detectable as in the single-exon PCR amplifications. The individual sequences derived from the two different PCR methods were compared at the level of individual signalto-noise $(\mathrm{S} / \mathrm{N})$ ratios of the four bases $A, C, G$, and $T$, as well as the proportion of high-quality (>99\% confidence scores obtained with KB base caller software; Applied Biosystems, Rotkreuz, Switzerland) basesignals.

For the amplification of single exons, PCR reactions were performed in hot-start wax tubes. Lower reaction mixtures were prepared containing $10 \times \mathrm{PCR}$ buffer $+\mathrm{Mg}^{2+}, \mathrm{PCR}$ nucleotide mix, and forward and reverse primers (each $10 \mu \mathrm{M}$ ). Upper mixes were prepared containing $1.85 \mathrm{U}$ high-fidelity Taq polymerase (Roche Diagnostics, Rotkreuz, Switzerland), 10× PCR buffer $+\mathrm{Mg}^{2+}$. Finally, $200 \mathrm{ng}$ template DNA was added to each reaction. $P C R$ reactions were conducted as follows: denaturation at $94^{\circ} \mathrm{C}$ for 5 min (MCAD exon $1,96^{\circ} \mathrm{C}$ ); 35 cycles at $94^{\circ} \mathrm{C}$ for $30 \mathrm{~s}$, annealing for $60 \mathrm{~s}$, and at $72^{\circ} \mathrm{C}$ for $90 \mathrm{~s}$; final extension at $72^{\circ} \mathrm{C}$ for $5 \mathrm{~min}$; annealing temperatures were $60^{\circ} \mathrm{C}$ for $M C A D$ exon 1 , $57^{\circ} \mathrm{C}$ for $M C A D$ exons $2-12$, and $55^{\circ} \mathrm{C}$ for all OTC exons.

For the amplification of the exons in multiplex PCR set-up, all reactions were performed with a multiplex PCR kit (Qiagen, Hombrechtikon, Switzerland, Cat\# 206143). Reaction mixtures were prepared containing $12.5 \mu \mathrm{L} 2 \times$ multiplex master mix, $2.5 \mu \mathrm{L} 10 \times$ primer mix (according to Table 1, each primer $2 \mu \mathrm{M}$ ), $2.5 \mu \mathrm{L}$ Q-solution, $4.5 \mu \mathrm{L}$ molecular grade water, and $3 \mu \mathrm{L}$ genomic DNA as template (up to $300 \mathrm{ng}$ ). PCR reactions were conducted as follows: denaturation at $95^{\circ} \mathrm{C}$ for $15 \mathrm{~min} ; 35$ cycles at $94^{\circ} \mathrm{C}$ for $30 \mathrm{~s}, 57^{\circ} \mathrm{C}$ (MCAD) or $55^{\circ} \mathrm{C}\left(\right.$ OTC) for $90 \mathrm{~s}$, and $72^{\circ} \mathrm{C}$ for $60 \mathrm{~s}$; final extension
Table 1 Composition of primer mixes for MCAD and OTC multiplex PCR reactions.

\begin{tabular}{lcl}
\hline MCAD mix & Exon \# & Fragment length (bp) \\
\hline 1 & 1 & 372 \\
& 2 & 300 \\
& $3 / 4$ & 426 \\
2 & 9 & 347 \\
& 5 & 319 \\
& 6 & 351 \\
3 & 10 & 293 \\
& 12 & 441 \\
& 7 & 315 \\
OTC mix & 8 & 291 \\
1 & 11 & 435 \\
& Exon \# & Fragment length (bp) \\
& 1 & 294 \\
& 3 & 223 \\
2 & 5 & 272 \\
& 7 & 183 \\
& 9 & 280 \\
& 2 & 246 \\
& 4 & 223 \\
& 6 & 213 \\
& 8 & 282 \\
\end{tabular}

at $72^{\circ} \mathrm{C}$ for $10 \mathrm{~min}$. A $5-\mu \mathrm{L}$ aliquot of the PCR products was analyzed on $1.5 \%$ agarose gel to verify the size of all products (Figure $1 \mathrm{~A}$ and $\mathrm{B}$ ).

For sequencing, all PCR products (single exon reactions or multiplex reactions) were directly purified with a PCR purification kit (Qiagen, Cat\# 28106). Individual PCR products of the multiplex PCR reactions were not separated for sequencing, thus the multiplex PCR products containing up to five different amplicons were directly used as templates for sequencing. For cycle sequencing, reaction mixtures were prepared as follows: $3.5 \mu \mathrm{L} 2.5 \times$ sequencing buffer, $0.5 \mu \mathrm{L}$ ready reaction mix (both from BigDye Terminator v3.1 cycle sequencing kit; Applied Biosystems, Cat\# 4336917), $1 \mu \mathrm{L}$ primer (5 $\mu \mathrm{M}), \mathrm{PCR}$ product (20-30 ng), and molecular grade water up to $10 \mu \mathrm{L}$ final volume. Cycle sequencing conditions were: denaturation at $96^{\circ} \mathrm{C}$ for $60 \mathrm{~s}, 25$ cycles at $96^{\circ} \mathrm{C}$ for $10 \mathrm{~s}$, at $50^{\circ} \mathrm{C}$ for $5 \mathrm{~s}$, and $60^{\circ} \mathrm{C}$ for $75 \mathrm{~s}$. Each cycle sequencing reaction was supplemented by $10 \mu \mathrm{L}$ molecular grade water and $2 \mu \mathrm{L} 2.2 \%$ sodium dodecyl sulfate and was denatured for $5 \mathrm{~min}$ at $98^{\circ} \mathrm{C}$. After purification on DyeEx columns (Qiagen, Cat\# 63206), sequences were separated on $A B \mid 3130 X L$ using POP7 polymer on a $50-\mathrm{cm}$ array, acquired using sequence detection software v.3.0 and analyzed with sequence analysis software v.5.2 (all from Applied Biosystems).

A total of 66 MCAD sequences (3 donors, 11 PCR products, forward and reverse sequences) obtained from multiplex PCR were compared with the corresponding sequences obtained from single-exon PCR. Out of 66 sequences, 56 derived from multiplex PCR products had higher $\mathrm{S} / \mathrm{N}$ ratios than the corresponding single-exon PCR derived sequences. The proportion of high-quality (>99\%) signals of the multiplex PCR derived sequences was significantly higher than that of the single-exon sequences $(97.73 \% \pm 1.8 \%$ vs. 
A
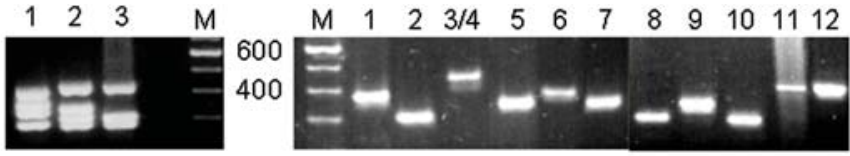

B
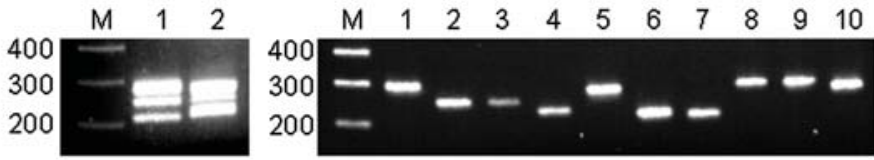

C

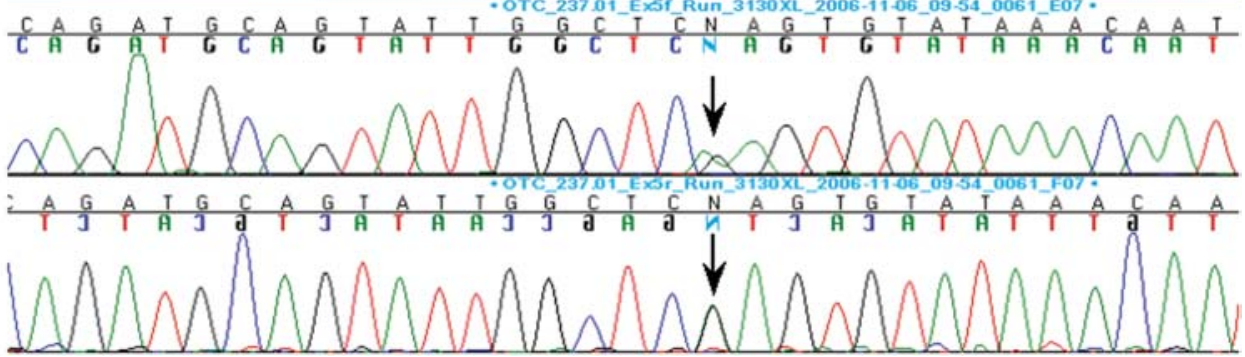

Figure 1 Gel-electrophoresis analyses of multiplex PCR and single-exon PCR.

PCR products were separated on $1.5 \%$ agarose gels, stained with ethidium bromide. (A) Multiplex PCR mixes 1-3 of MCAD (left) and single-exon PCR products 1-12 (right), as indicated. Fragment sizes of a 100-bp DNA ladder (M) are indicated. The fragment lengths of the PCR products are listed in Table 1. (B) Multiplex PCR mixes 1 and 2 of OTC (left) and single-exon PCR products 1-10 (right), as indicated. (C) Screenshot of a multiplex PCR derived sequence. Upper panel, forward sequence; lower panel, reverse sequence; the heterozygous mutation is indicated by an arrow.

$95.92 \% \pm 3.8 \% ; \mathrm{p}<0.0001 ;$ non-parametric two-tailed signed-rank Wilcoxon test). The range of the proportion of high-quality signals was $91.14 \%-100 \%$ for multiplex sequences, and $76.9 \%-99.52 \%$ for singleexon sequences, respectively. In the case of MCAD analysis, the switch to multiplex PCR did not only facilitate the experimental work process, but also even more importantly, improved the sequence quality.

In contrast to these results, only 16 out of a total of 98 sequences derived from multiplex PCR of the OTC exons had equal or higher $\mathrm{S} / \mathrm{N}$ ratios than the corresponding single-exon derived sequences. A total of 98 OTC sequences (5 donors, 10 exons, forward and reverse, except exon 1, only 3 donors) generated by multiplex and single-exon PCR, respectively, were included in this analysis. Even though the proportion of high-quality scores of the multiplex PCR derived sequences was slightly lower $(94.32 \% \pm 3.3 \%$ vs. $94.87 \% \pm 4.8 \% ; p=0.017$; non-parametric two-tailed signed-rank Wilcoxon test), the diagnostic power of this test was not affected. First, the mutations bearing a phenotypic consequence are not located at the beginning or the end of the amplicons, where some ambiguous bases might occur in the multiplex sequences. Secondly, these analyses are based on genomic DNA, thus all mutations are either homozygous (in males) or heterozygous (females), and in the latter case the bases of both alleles are represented with equal signal strength (Figure 1C).

Even though the improvement of signal quality cannot always be achieved, the switch from single-exon PCR to multiplex PCR is still reasonable, as I) the workload and costs of PCR and purification of PCR products are markedly reduced, and II) the risk of pipetting errors is reduced, as primer master mixes can be prepared. Once tested, these master mixes can be stored in aliquots for several months. In emergency cases, as is often the case, when newborns show symptoms of MCAD or OTC deficiency, the sequence analysis of all exons might be performed in less than $48 \mathrm{~h}$, including DNA extraction, multiplex PCR, cycle sequencing and sequence analysis. Furthermore, it is worth mentioning that a higher degree of multiplexing may be achieved for these two genes using the proposed protocol even without re-designing primer pairs. However, the combinations of exons in the different PCR reactions were chosen to allow a visual control of the PCR amplification by agarose gel electrophoresis (Figure $1 \mathrm{~A}$ and $\mathrm{B}$ ).

Because we have not made any specific re-design of the PCR primers for multiplexing of the OTC and $M C A D$ genes and because we could successfully apply a standard PCR protocol to both genes without any optimization steps, we suggest that many existing sequencing based mutation analysis protocols could be easily optimized with the proposed method for many other genes, in which the mutations known and so-far unknown - can be distributed over the whole genes. Indeed, we successfully established multiplex PCR using the described protocol for mutation screening by DNA sequencing in the proto-oncogene p53, as well as other multi-exon genes, not reported here.

\section{Acknowledgements}

Financial support for this work was provided by a research grant from the Swiss National Science Foundation (31119839) to C.R.L. 


\section{References}

1. Waddell L, Wiley V, Carpenter K, Bennetts B, Angel L, Andresen BS, et al. Medium-chain acyl-CoA dehydrogenase deficiency: genotype-biochemical phenotype correlations. Mol Genet Metab 2006;87:32-9.
2. Yamaguchi S, Brailey LL, Morizono $H$, Bale AE, Tuchman $M$. Mutations and polymorphisms in the human ornithine transcarbamylase (OTC) gene. Hum Mutat 2006;27:62632.

3. Henegariu O, Heerema NA, Dlouhy SR, Vance GH, Vogt $\mathrm{PH}$. Multiplex PCR: critical parameters and step-by-step protocol. Biotechniques 1997;23:504-11. 